\title{
Analysis of Dynamic Changes in ECG Signals during Optical Mapping by Dynamic Time Warping
}

\author{
M Vyklicky ${ }^{1}, \mathrm{~J} \mathrm{Bardonova}^{1}$, I Provaznik ${ }^{1}$, M Novakova ${ }^{2}$, \\ M Blaha ${ }^{1}$, J Kozumplik $^{1}$ \\ ${ }^{1}$ Brno University of Technology, Brno, Czech Republic \\ ${ }^{2}$ Masaryk University in Brno, Brno, Czech Republic
}

\begin{abstract}
Voltage-sensitive dyes (VSDs) are used in touch-less recording of electrical activity from animal hearts. Previously, VSDs has been successfully used in myocardial ischemia studies. However, our previous Langendorff perfused heart experiments have suggested changes in ECG signals in time and time-frequency domain caused by application of VSD during optical mapping. Detailed analysis of changes is needed to reveal impact to particular fractions of a heart cycle. Dynamic time warping $(D T W)$ is applied to $P-Q$ segments, $Q R S$ complexes, and ST-T segments of selected consecutive heart cycles in each experiment period. An optimal path resulting from DTW based on minimum distance between the control segments (before the application of VSD) and segments recorded in next two experiment steps is computed.
\end{abstract}

\section{Introduction}

Ischemic heart disease (or coronary h.d.), the most common cause of death in the industrialized countries [1], covers a range of conditions in which the blood supply to the heart muscle, and consequently the heart functions, become limited. As a rule, it is caused by a narrowing of a branch of the coronary artery (stenosis) by atherosclerotic process.

The diagnostic processes of ischemic heart disease consist of invasive and non-invasive methods. The development of non-invasive methods has been required by the tendency to avoid the untoward side effects during instrumentation, risk of iatropathogenic damage (mainly by infection), and last but not least, the economic aspects of cardiovascular diagnostics. Further, the results could help to develop a new noninvasive method needed in cardiology diagnostics.

Ischemic heart disease is instrumentally diagnosed by coronary angiography, which represents the reference method. Echocardiographic data offer valuable information on regional or global impairment of cardiac function as do the radionuclid perfusion methods based on thallium 201 or technetium 99 uptake. For more than a half of a century, the most widely used diagnostic test for the detection and evaluation of cardiac ischemia are the electrocardiographic method [2]. The attenuated or stopped coronary perfusion causes a potential difference between the ischemic and normal regions during the ST segment and, eventually, also at rest. While ST depression is the common manifestation of transitory, exercise-induced cardiac ischemia, ST elevation is related to severe transmural ischemia, acute myocardial infarction. Myocardial ischemia affects also other features than the ST segment [3] including an increase of QRS amplitude, subtle prolongation of QRS duration and shifts of QRS axis [4]. These changes reflect abnormal conduction due to irregular membrane depolarization. There is ample evidence that cardiac ischemia may change the QRS spectrum as the expression of fragmentation of ventricular depolarization.

The recent findings show that acute myocardial ischemia in early stages can be detected using the analysis of intra-QRS changes. These changes reveal local ischemia-induced propagation changes earlier than traditional ECG-based indexes [5], [6]. Recent findings specify the changes as low-amplitude short-time events within a window of ventricular depolarization. The actual time and frequency localization of the changes depend on the heart status.

Myocardial ischemia studies using optical mapping consists of four experimental phases: control period, dye loading, wash-out, myocardial ischemia. The use of VSDs may negatively influenced electrophysiology of the examined heart. Therefore, dynamic changes in electrocardiograms during particular phases of the experiment must be studied.

A number of method based on deterministic or stochastic theory can be applied. One of the possibility is analysis of time-frequency spectra of the signals. Timefrequency analysis reveals changes in signals with optimum time- and frequency resolution. Thus, it can help to detect low level and short-time changes in the 
same time. However, time-frequency spectrum is complex parameter and its further analysis is complicated. Regarding variability in length of particular ECG signal segments during the experiment, dynamic time warping (DTW) applied to parts of time-frequency spectra seems to be on of the suitable mathematical tools.

\section{Methods}

The Ethical Committee of the Masaryk University Brno approved the experimental protocol used in this study. Four rabbits under deep ether anesthesia were sacrificed. The chest was quickly opened, the heart with sufficiently long piece of aorta immediately cut-off and placed in a preparation bowl with a cold $\left(5^{\circ} \mathrm{C}\right)$ KrebsHenseleit $(\mathrm{K}-\mathrm{H})$ solution $\left(1.2 \mathrm{mM} \mathrm{Ca}^{2+}\right)$. The aorta was cannulated and the heart perfused at the constant perfusion pressure $(100 \mathrm{mmHg})$ at the temperature $37^{\circ} \mathrm{C}$. After stabilization period $(15-20 \mathrm{~min})$, the isolated heart was loaded with voltage-sensitive dye for 20 minutes and washed-out with $\mathrm{K}-\mathrm{H}$ solution for 15 minutes. Then the preparation was ready for measurements, which took 60 minutes. During all experiment, simultaneous touch-free recordings of electrogram were done.

The employed optical recording system is based on application of voltage sensitive dye di-4-ANEPPS (Molecular Probes, USA) into the examined tissue [7]. The dye undergo changes in its fluorescence spectra, in response to changes in the surrounding electric field.

The ECG signals from orthogonal leads were recorded from $\mathrm{Ag}-\mathrm{AgCl}$ electrodes positioned on the inner surface of the bath. The signals were digitized by a 12-bit AD converter at $4 \mathrm{kHz}$ sampling rate using a data acquisition multifunction card PCI-6111E (National Instruments, USA). The digital signal was stored on a hard disk for further off-line processing.

\section{Data processing}

Recorded data was pre-processed by wavelet-based Wiener filter for ECG signal denoising [8]. Then data was downsampled from $4000 \mathrm{~Hz}$ to $500 \mathrm{~Hz}$. An example of a recorded ECG signal sampled at $4 \mathrm{kHz}$ is on panel a) of Fig.1. Panel b) of the same figure shows a Wiener filtered and downsampled signal from panel a).

Data was divided for P-Q segments, QRS complexes, and ST-T segments of selected consecutive heart cycles in each experiment period. Than, wavelet transform and dynamic time warping was applied as described below.
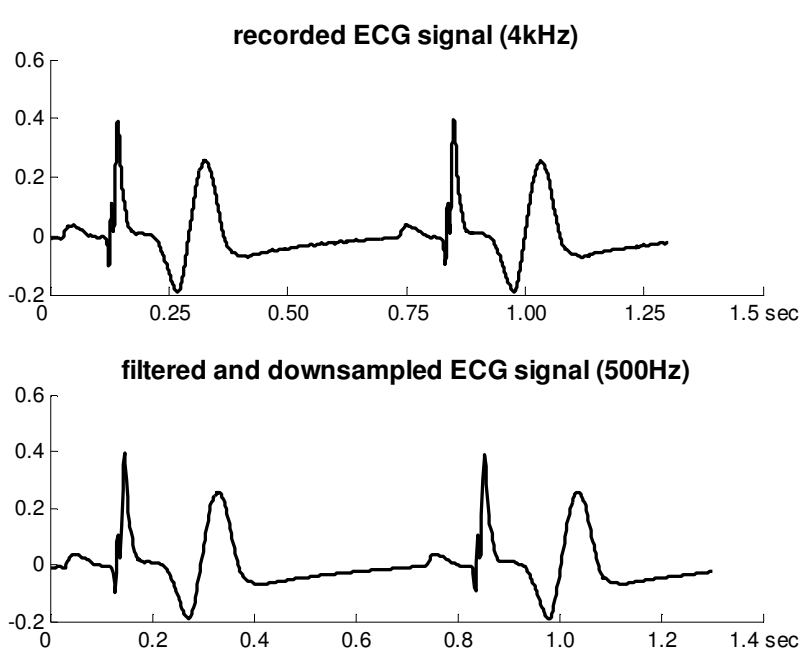

Figure 1. a) A recorded ECG signal. b) Down-sampled and Wiener filtered signal from panel a).

\subsection{Wavelet transform}

A common mathematical tool for time-frequency analysis is short-time Fourier transform (STFT). STFT is Fourier transform applied to a windowed signal while the window is shifted in time. Constant width of the window causes low time resolution in various frequency bands. To overcome this problem, wavelet transform (WT) can be used. WT uses a basis of functions of two parameters: time shift and time dilation. To decompose the analysed signal, projection to each dilated basis function (wavelet) must be computed. WT is then defined as correlation of signal $\mathrm{x}(\mathrm{t})$ with wavelets $\mathrm{g}^{*}[(\mathrm{t}-\tau) / \lambda]$, where $\tau$ is time shift, $\lambda$ is time dilation, and $*$ represents complex conjugate:

$$
C W T(\lambda, \tau)=\int_{-\infty}^{\infty} \frac{1}{\sqrt{\lambda}} \mathrm{g} *\left(\frac{\mathrm{t}-\tau}{\lambda}\right) \mathrm{x}(\mathrm{t}) \mathrm{dt}
$$

For the time-frequency analysis, Morlet wavelet has been used for its relatively smooth shape [9]. The analysis resulted in a sequence of vectors representing frequency components between $0.01-250 \mathrm{~Hz}$ at each time instant. The vectors were used as time sequences for DTW as described below.

\subsection{Dynamic time warping in ECG signal processing}

Dynamic time warping is a method which calculates distance between two time sequences (signals) and searches for a single optimal sequence. A component of the first signal has its corresponding counterpart in the second signal but may be distorted in time (dilated, 
compressed, shifted, etc.). This also applies in the case of the signals with different lengths. DTW works with nonlinear time normalization. Moreover, the normalization can be limited by specific parameters to prohibit large fluctuation in a time domain model. Time differences between two time sequences are eliminated by warping of time axes. This problem is formulated as a maximum likelihood path searching optimisation process [10], [11].

In the proposed algorithm, sequences of frequency spectra are used as time sequences. These frequency spectra are extracted from time-frequency spectrum, which was calculated by wavelet transform for each ECG signal (see Fig.2 a) and Fig.2 b) [12].

Consider two time sequences $A$ and $B$, of length $I$ and $J$ respectively, where:

$$
\begin{aligned}
& A=\{a(1), a(2), \ldots, a(n), \ldots, A(I)\} \\
& B=\{b(1), b(2), \ldots, b(m), \ldots, b(J)\}
\end{aligned}
$$

The algorithm with DTW searches for optimal path $m=\psi(n)$ in the plane $(n, m)$, which minimizes a function $D$. The function $D$ is computed as overall distance between time sequences $\mathrm{A}$ and $\mathrm{B}$ as

$$
D(A, B)=\sum_{n=1}^{I} d[a(n), b(\psi(n))]
$$

where $d[a(n), b(\psi(m))]$ is local distance between $n$-th element of $A$ and $m$-th element of $B$.

Optimal path $m=\psi(n)$ expresses relation between $m$ and $\mathrm{n}$ by a simple function. Suppose a general time variable $k$, both time variables $m$ and $n$ can be expressed as a function with parameter $k$ as, $n=i(k), m=j(k)$ for $k=1, \ldots, K$, where $K$ is length of general time axis for comparison of sequences $A$ and $B$, reference sequence and test sequence especially.

In this case, dynamic time warping (DTW) is applied to time-frequency spectrum of P-Q segments, QRS complexes, and ST-T segments of selected consecutive heart cycles in each experiment period, namely control period, loading of VSD, washout and ischemia. An optimal path resulting from DTW based on minimum distance between the control segments (before the application VSD) and segments recorded in next two experiment steps is computed. The length of discussed segments was significantly different, namely the length of ST-T segment ranged around $0.6 \mathrm{sec}$ and P-Q segment in the same heart cycle around 0.05 sec. To decrease effect of the length, the final distance between two vector sequences was divided by length of DTW optimal path

$$
D=D(A, B) / K
$$

The example of optimal path DTW is shown on Fig. 2 c).
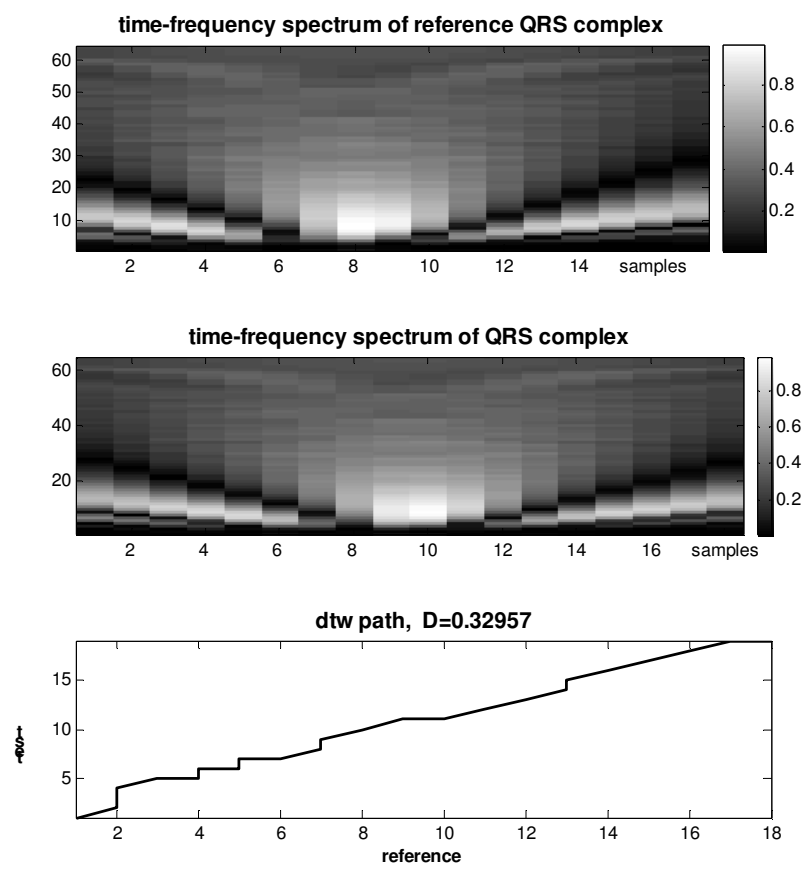

Figure 2. a) Time-frequency spectrum of reference QRS complex, recorded before loading VSD; b) Timefrequency spectrum of QRS complex compared with reference's one; c) Progress of DTW optimal path - Xaxis correspond to the length of reference sequences of vectors, taken from time-frequency spectrum of control period of ECG, y-axis correspond to the length of test sequences of vectors, taken from time-frequency spectrum of ECG.

\section{Results and discussion}

The proposed algorithm based on dynamic time warping has been tested on ECG signals regarding application of voltage-sensitive dyes. Generally, significant changes in the optimal path computed according to Eq. 3 has been observed during the VSD loading. In the end of wash-out period, changes in the optimal path were considerably lower for all segments or they at least had decreasing trend. As expected, optimal path was remarkably different in myocardial ischemia period due to known electrophysiological effect of coronary artery occlusion, see Fig. 3.

It may be concluded that optimal path of dynamic time warping applied to ECG signals is sensitive to loading of VSD into examined heart. It can distinguish particular periods of optical mapping procedure. Further, DTW can be used to analyse various fractions of a heart cycle and thus be exploited for analysis of VSD influence to the conductive system of the heart. 


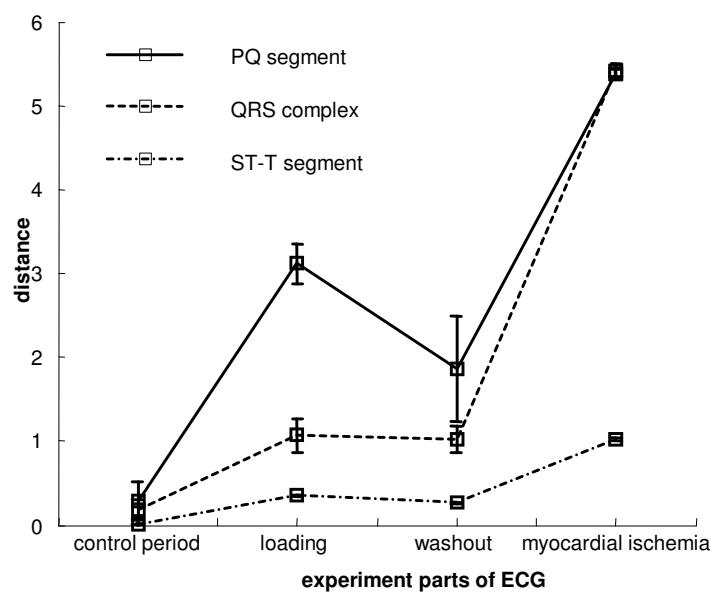

Figure 3. Variability of distance between representative control ECG cycle and cycles during experiments. Solid variability of P-Q segments; dashed - variability of QRScomplexes; dashdotted - variability of ST-T segments.

\section{Acknowledgements}

The research was supported by the grants 102/04/0472 and 305/04/1385 from GACR, and by Research Programme of Brno University of Technology MSM 0021630513 .

\section{References}

[1] Alpert JS, Thygesen K. Myocardial infarction redefined. J Am Coll Cardiol 2000; 36(3):959-969.

[2] Drew BJ, Krucoff MW. Multilead ST-segment monitoring in patients with acute coronary syndromes: a consensus statement for healthcare professionals. Am J Critical Care 2000; 8(6):372-386.

[3] Kligfield P. ST segment analysis in exercise stress testing. In: Zareba W, Maison-Blanche P, Locati EH. Noninvasive Electrocardiology in Clinical Practice, Futura Publishing Co. 2001.
[4] Lemire D, Pharand C, Rajaonah J-C, Dube B, LeBlanc AR. Wavelet time entropy, $\mathrm{T}$ wave morphology and myocardial ischemia. IEEE Trans Biomed Eng 2000; 47(7):967-970.

[5] Bardonova J, Provaznik I, Novakova M, Novakova Z. Hidden Markov Model in Wavelet Analysis of Myocardial Ischemia in Rabbit. Computers in Cardiology 2000:419422.

[6] Gramatikov B, Brinker J, Yi-chun S, Thakor NV. Wavelet Analysis and Time-Frequency Distributions of the Body Surface ECG Before and After Angioplasty. Computer Methods and Programs in Biomedicine 2000; 62:87-98.

[7] Provaznik I, Novakova M, Vesely Z, Blaha M, Chmelar M. Electro-optical recording system for myocardial ischemia studies in animal experiments. Computers in Cardiology 2003; 30:573-576.

[8] Chmelka L, Kozumplik J. Wavelet-based Wiener filter for electrocardiogram signal denoising. Computers in Cardiology 2005, to be printed.

[9] Aldroubi A, Unser M. Wavelets in Medicine and Biology. CRC Press, New York, 1996.

[10] Garcia J, Wagner G, Sornmo L, Laguna P. Identification of the Occluded Artery in Patients with Myocardial Ischemia Induced by Prolonged Percutaneous Transluminal Coronary Angioplasty Using Traditional vs Transformed ECG-Based Indexes. Comp Biomed Res 1999; 32:470-482.

[11] Gollmer K, Posten C. Supervision of Bioprocesses Using a Dynamic Time Warping Algorithm. Control Eng. Practise 1996; 4(9):1287-1295.

[12] Bardonova J, Provaznik I. Novakova M, Novakova Z. Application of Dynamic Time Warping in ECG Description for Recognition of Myocardial Ischemia. Biosignal 2002; 16:236-239.

Address for correspondence.

Jana Bardonova

Department of Biomedical Engineering

Brno University of Technology

Kolejni 4

61200 Brno

Czech Republic 